\title{
Active Disturbance Rejection Approach for Robust Fault-Tolerant Control via Observer Assisted Sliding Mode Control
}

\author{
John Cortés-Romero, ${ }^{1}$ Harvey Rojas-Cubides, ${ }^{1}$ Horacio Coral-Enriquez, ${ }^{1}$ \\ Hebertt Sira-Ramírez, ${ }^{2}$ and Alberto Luviano-Juárez ${ }^{3}$ \\ ${ }^{1}$ Departamento de Ingeniería Eléctrica y Electrónica, Universidad Nacional de Colombia, Carrera 45 No. 26-85, Bogotá, Colombia \\ ${ }^{2}$ Centro de Investigación y de Estudios Avanzados del Instituto Politécnico Nacional (CINVESTAV-IPN), \\ Avenida Instituto Politécnico Nacional, No. 2508, Departamento de Ingeniería Eléctrica, Sección de Mecatrónica, \\ Colonia San Pedro Zacatenco A.P. 14740, 07300 México, DF, Mexico \\ ${ }^{3}$ Unidad Profesional Interdisciplinaria en Ingeniería y Tecnologías Avanzadas (UPITTA), Instituto Politécnico Nacional, \\ Avenida Instituto Politécnico Nacional 2580, Barrio La Laguna Ticomán, Gustavo A. Madero 07340, México, DF, Mexico \\ Correspondence should be addressed to John Cortés-Romero; jacortesr@unal.edu.co
}

Received 23 August 2013; Revised 4 November 2013; Accepted 6 November 2013

Academic Editor: Baoyong Zhang

Copyright (C) 2013 John Cortés-Romero et al. This is an open access article distributed under the Creative Commons Attribution License, which permits unrestricted use, distribution, and reproduction in any medium, provided the original work is properly cited.

This work proposes an active disturbance rejection approach for the establishment of a sliding mode control strategy in faulttolerant operations. The core of the proposed active disturbance rejection assistance is a Generalized Proportional Integral (GPI) observer which is in charge of the active estimation of lumped nonlinear endogenous and exogenous disturbance inputs related to the creation of local sliding regimes with limited control authority. Possibilities are explored for the GPI observer assisted sliding mode control in fault-tolerant schemes. Convincing improvements are presented with respect to classical sliding mode control strategies. As a collateral advantage, the observer-based control architecture offers the possibility of chattering reduction given that a significant part of the control signal is of the continuous type. The case study considers a classical DC motor control affected by actuator faults, parametric failures, and perturbations. Experimental results and comparisons with other established sliding mode controller design methodologies, which validate the proposed approach, are provided.

\section{Introduction}

The main challenge of the Fault-Tolerant Control is to guarantee high performance and reliability in the most adverse operations such as the presence of perturbations, disturbances, dynamic miss-modeling, and actuator faults among others. In general, the techniques employed on the Fault-tolerant Control (FTC) can be classified into active and passive. Active FTC is characterized by the controller reconfiguration assisted by fault detection and isolation (FDI) schemes [1]. On the other hand, passive techniques exploit the robustness of some types of controllers without requiring changes in their structure and can operate satisfactorily without information about system failures. These techniques are usually simple in implementation but are not usually suitable for severe cases of failures [1].

The robust characteristics of the sliding mode technique provide a natural environment for the use of such techniques on passive FTC schemes. This technique has been properly used in different control schemes and assisted by other effective control strategies which have shown proper performance under fault-tolerant operations (see [1-4] as representative examples). Over the past years, considerable attention has been paid to the design of linear/nonlinear disturbance observers for sliding mode controller assistance in order to overcome several issues like chattering [5-8], disturbances and system uncertainties [8-10], coupling of MIMO systems [11], or uncertainties, disturbances, and actuator faults [12]. 
Even though the performance of the aforementioned control proposals is accurate, there are still complexities in the design that are a consequence of dealing with the system faults and disturbances separately, on the one hand, and, on the other hand, the need for precise knowledge of the system model.

In the active disturbance rejection control (ADRC) philosophy, system fault and disturbances can be dealt with unitedly rendering a simplified linear control structure based on a simplified model like the classical passive fault-tolerant scheme. From the ADRC point of view, the disturbances must be rejected in an active manner, so the control system actively produces accurate estimates and reduces the causes of the output errors. ADRC as a potential solution has been explored in several domains of control engineering (see [1315]). In accordance with this field, Generalized Proportional Integral (GPI) observers were introduced in [16]. Despite of grand ADRC applications reported in the literature, the potential of this technique for fault-tolerant performance has been scarcely considered. Under the ADRC setup, a GPI disturbance observer assisted sliding mode control approach can be used to deal with fault-tolerant operation. In this paper, the linear GPI observers are used as a part of an active disturbance rejection scheme for the sliding mode creation problem on nonlinear systems with low switching authority.

We are interested in a proper local sliding mode creation with the aid of a GPI disturbance observer. In the establishment of the slide surface, unknown inputs (state dependent or external) impact the correct evolution of the sliding regime demanding greater bound of the control input; when the sliding surface dynamics include an active disturbance cancellation of the influence of that kind of unknown inputs, the required switching input amplitude can be decreased. Furthermore, risk for deviations from the sliding surface, due to unexpected control input saturations, is practically avoided. The proposed GPI observer can be related to either the system dynamics or sliding surface dynamics disturbance inputs; in both cases, it is possible to correctly design a suitable assisted sliding mode control law with fault-tolerant capabilities.

It is assumed that the effect of additive state-dependent and exogenous nonlinearities, that affect the sliding mode regime, may be approximately but accurately canceled from the nonlinear system behavior via the injection of a precise and exogenously generated time-varying signal.

In this work we propose an approach of passive faulttolerant control based on a classic sliding mode controller assisted by a GPI observer under the context of the active disturbance rejection. This scheme has been validated with the control of a DC motor subject to perturbations in the load torque, actuator faults, and parametric failures.

This paper is organized as follows. Section 2 explores the possibilities of the ADRC in GPI based observer sliding mode control for fault-tolerant operation and two related useful cases are presented. Section 3 describes the study case, states the formulation of the problem, and presents its corresponding proposed design. Section 4 is devoted to the presentation of the experimental results describing experimental platform and the experiments that were carried out to enhance the advantages of using the linear estimation of the disturbance functions during the sliding mode creation problem. Finally, Section 5 contains the conclusions.

\section{Possibilities of ADRC for Sliding Mode Control Assistance}

It is possible to assist the creation of a sliding mode regime for a wide variety of sliding mode control strategies. The idea is to inject a continuous term via a suitably defined observer, in an active fashion, at the controller stage to ensure the correct establishment or continuation of the sliding mode regime.

The objective of the proposed fault-tolerant control design is to accurately track a desired reference trajectory, even in the presence of the unknown disturbances caused by actuator faults, parameter uncertainty, the presence of unmodeled state-dependent nonlinearities, or the combination of these previous cases with the presence of uncertain exogenous time-varying signals.

This is explained in this section by using a GPI observerbased sliding mode controller. From this point of view, all those terms are considered as a single, lumped, unstructured, time-varying disturbance term. In the establishment of the sliding mode control law, it is necessary to have an estimation of the related disturbance term. Two main benefits of using this strategy can be highlighted: (1) GPI observers allow the estimation of the state of the system, the related disturbance function, and a certain number of its time derivatives; (2) the control law is composed of a discontinuous term plus a continuous injection provided by the GPI observer. The amplitude of the switching part $(W)$ acts as a weighting factor allowing the chattering reduction.

In the following section, two approaches for the creation of the sliding mode regimes assisted by GPI observers, suitable for fault-tolerant operation, are explained.

It should be noted that our approach is not the only possibility; it is merely a preferred approach with ease of analysis, (e.g., it is possible to propose a GPI observer assisted strategy of high-order sliding mode).

2.1. On Observer Assisted First Order Sliding Mode Creation. In this subsection a conventional first order sliding mode control is appropriately adapted by a GPI observer. Consider the following $n$-dimensional, nonlinear, single-input singleoutput system:

$$
\dot{x}=f(x)+g(x) u, \quad \sigma=h(x),
$$

where the drift vector field $f(x)$ is a smooth but uncertain vector field on $T \mathbb{R}^{n}, g(x)$ is known and a smooth vector field on $T \mathbb{R}^{n}$, and $u$ is the control input taking values on the closed interval $[-U, U], U>0$. The function $h(x)$ is a smooth function $h: \mathbb{R}^{n} \rightarrow \mathbb{R}$. The zero level set for the scalar output $\sigma$

$$
S=\left\{x \in \mathbb{R}^{n} \mid \sigma=h(x)=0\right\},
$$

represents a smooth, $n-1$ dimensional manifold acting as a sliding surface, where $\sigma$ is the sliding surface coordinate function. 
The state-dependent unperturbed sliding surface dynamics are characterized by

$$
\dot{\sigma}=L_{f} h(x)+L_{g} h(x) u,
$$

where $L_{f}$ and $L_{g}$ are the Lie derivatives or the directional derivatives of $h$, along the directions of the vectors $f$ and $g$, respectively.

Actuator faults, exogenous disturbances, modeled and non modeled internal dynamics, and possible parameter variation can be treated as an equivalent additive lumped disturbance function, $\xi_{\sigma}$, affecting the sliding surface dynamic $\sigma$ :

$$
\dot{\sigma}=\xi_{\sigma}+L_{g} h(x) u
$$

\subsubsection{Assumptions}

Assumption 1. The amplitude, $W$, of the switching part of the control input $u$ satisfies $W<U$.

Assumption 2. The disturbance function, $\xi_{\sigma}$, and a finite number of its time derivatives, $\xi_{\sigma}^{(k)}, k=0,1,2, \ldots, m$, for a sufficiently large $m$, are assumed to be uniformly and absolutely bounded; that is, $0 \leq\left|\xi_{\sigma}^{(k)}\right| \leq \delta_{k}<\infty$ for any feedback control input stabilizing the sliding surface coordinate dynamics.

Assumption 3. We assume that $L_{g} h(x)>0$ is perfectly known and locally strictly positive.

The key observation for the robust operation of the proposed sliding regimes is based on the accurate, yet approximate, on-line estimation of the scalar uncertain disturbance function $\xi_{\sigma}$ in the form $\widehat{\xi}_{\sigma}$. The incorporation of that estimation in the sliding mode control law may result in a substantially enhanced possibility for the creation of a sliding motion via the active disturbance cancelation strategy:

$$
u=\frac{1}{L_{g} h(x)}\left[-\widehat{\xi}_{\sigma}-W \operatorname{sign}(\sigma)\right]
$$

An extended state representation can be proposed to cope with the disturbance function estimation $\widehat{\xi}_{\sigma}$. The augmented representation is based on the internal model of the disturbance function $\xi_{\sigma}$. When there is no previous knowledge about the disturbance term $\xi_{\sigma}$, a general signal oriented approach can be quite effective for on-line estimation purposes. Associated with ADRC and specialized by GPI approaches, unknown input signals can be approximated by $d^{m} \xi_{\sigma} / d t^{m} \approx 0$. For that realization, the extended state vector

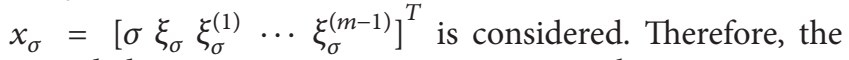
extended state space representation is given by

$$
\dot{x}_{\sigma}=A_{1} x_{\sigma}+B_{\sigma} L_{g} h(x) u+E_{\sigma} \xi^{(m)},
$$

where

$$
A_{1}=\left[\begin{array}{ccccc}
0 & 1 & 0 & \cdots & 0 \\
0 & 0 & 1 & & 0 \\
\vdots & & & \ddots & \vdots \\
0 & 0 & 0 & \cdots & 1 \\
0 & 0 & 0 & \cdots & 0
\end{array}\right], \quad B_{\sigma}=\left[\begin{array}{c}
1 \\
0 \\
\vdots \\
0 \\
0
\end{array}\right], \quad E_{\sigma}=\left[\begin{array}{c}
0 \\
0 \\
\vdots \\
0 \\
1
\end{array}\right] .
$$

The disturbance function estimation is given by the following GPI observer.

Theorem 1. Letting $z=\left[\begin{array}{llll}z_{1} & z_{2} & \cdots & z_{m+1}\end{array}\right]^{T}$ and $\Gamma=$ $\left[\begin{array}{llll}\gamma_{m} & \gamma_{m-1} & \cdots & \gamma_{0}\end{array}\right]^{T}$, with Assumptions 1-3, the following observer for system (4):

$$
\dot{z}=A_{1} z+B_{\sigma} L_{g} h(x) u+\Gamma e_{\sigma}
$$

with $m$ being a sufficiently large integer, produces exponentially asymptotic estimation of $\sigma, \xi_{\sigma}, \ldots, \xi_{\sigma}^{(m-1)}$ given by the observer variables $z_{1}, z_{2}, \ldots, z_{m}$, respectively. The estimation errors ( $\sigma-$ $\left.z_{1}\right),\left(\xi_{\sigma}-z_{2}\right), \ldots,\left(\xi_{\sigma}^{(m-1)}-z_{m+1}\right)$ are ultimately uniformly bounded given the design parameters $\gamma_{0}, \ldots, \gamma_{m}$ that are chosen so that the following characteristic polynomial is Hurwitz:

$$
p_{e_{\sigma}}(s)=s^{m+1}+\gamma_{m} s^{m}+\cdots+\gamma_{1} s+\gamma_{0} .
$$

Proof. The corresponding estimation error vector is defined as $\widetilde{e}_{\sigma}=x_{\sigma}-z$ and satisfies

$$
\dot{\tilde{e}}_{\sigma}=\left(A_{1}-L C\right) \widetilde{e}_{\sigma}+E_{\sigma} \xi^{(m)}=A_{\sigma} \widetilde{e}_{\sigma}+E_{\sigma} \xi^{(m)},
$$

with

$$
A_{\sigma}=\left[\begin{array}{ccccc}
-\gamma_{m} & 1 & 0 & \cdots & 0 \\
-\gamma_{m-1} & 0 & 1 & & 0 \\
\vdots & & & \ddots & \\
-\gamma_{1} & 0 & 0 & \cdots & 1 \\
-\gamma_{0} & 0 & 0 & \cdots & 0
\end{array}\right] \in \mathbb{R}^{(1+m) \times(1+m)}
$$

and its characteristic polynomial in the complex variable $s$ is given by

$$
p_{e_{\sigma}}(s)=\operatorname{det}\left(s I-A_{\sigma}\right)=s^{m+1}+\gamma_{m} s^{m}+\cdots+\gamma_{1} s+\gamma_{0},
$$

where the eigenvalues of $A_{\sigma}$ can be placed as desired by selecting the gain vector $\Gamma$. The Hurwitzian character of $A_{\sigma}$ implies that, for every constant, $(1+m) \times(n+m)$, symmetric, positive definite matrix $Q=Q^{T}>0$, there exists a symmetric, positive definite $(1+m) \times(1+m)$ matrix $P=P^{T}>0$, so that $A_{\sigma}^{T} P+P A_{\sigma}=-Q$. The Lyapunov function candidate $V(x)=$ $(1 / 2) e_{\sigma}^{T} P e_{\sigma}$ exhibits a time derivative, along with the solutions of the closed loop system given by

$$
\dot{V}\left(\widetilde{e}_{\sigma}, t\right)=\frac{1}{2} \widetilde{e}_{\sigma}^{T}\left(A_{\sigma}^{T} P+P A_{\sigma}\right) \widetilde{e}_{\sigma}+\widetilde{e}_{\sigma}^{T} P E_{\sigma} \xi_{\sigma}^{(m)}(t) .
$$

For $Q=I$, that is, an $(1+m) \times(1+m)$ identity matrix, this function satisfies

$$
\begin{aligned}
\dot{V}\left(x_{\sigma}, t\right) & =\frac{1}{2} \widetilde{e}_{\sigma}^{T}(-Q) \widetilde{e}_{\sigma}+\widetilde{e}_{\sigma}^{T} P E_{\sigma} \xi_{\sigma}^{(m)}(t) \\
& \leq \frac{1}{2}\left\|\widetilde{e}_{\sigma}\right\|_{2}^{2}+\left\|\widetilde{e}_{\sigma}\right\|_{2}\|P\|_{2}\|E\|_{2} \delta_{m} .
\end{aligned}
$$


Given that $\|E\|_{2}=1$ and according to Assumption 2, this function is strictly negative everywhere outside the sphere $S_{\sigma}$, given by

$$
S_{\sigma}=\left\{\widetilde{e}_{\sigma} \in R^{1+m} \mid\left\|\widetilde{e}_{\sigma}\right\|_{2} \leq 2 \delta_{m}\|P\|_{2}\right\}
$$

Hence, all trajectories $\widetilde{e}_{\sigma}(t)$ starting outside this sphere converge towards its interior, and all those trajectories starting inside $S_{\sigma}$ will never abandon it.

Corollary 2. Under all the previous assumptions, the discontinuous active disturbance rejection feedback controller

$$
u=\frac{1}{L_{g} h(x)}\left[-z_{1}-W \operatorname{sign}(\sigma)\right]
$$

locally creates a sliding regime for any amplitude, $W$, satisfying: $W>\delta_{0}$, with $\delta_{0}$ as the ultimate bound for the disturbance estimation error $e_{\sigma}$.

Proof. The observer-based control law renders the following closed loop sliding surface dynamics:

$$
\dot{\sigma}=\left(\xi_{\sigma}-\widehat{\xi}_{\sigma}\right)-W \operatorname{sign}(\sigma)
$$

with $\widehat{\xi}_{\sigma}=z_{1}$, which would require a smaller control input switching amplitude $W$ than in the case where the observer is not used. According to Theorem 1, the disturbance estimation error, $e_{\sigma}$, is bounded by $\delta_{1}$, and the local existence of a sliding regime $\sigma=0$ is guaranteed even if $W$ is rather small.

Consider the following Lyapunov function candidate:

$$
V=\frac{1}{2} \sigma^{2}
$$

Differentiating the Lyapunov function (18) with respect to time and using (17) yield

$$
\begin{gathered}
\dot{V}=\sigma \dot{\sigma}, \\
\dot{V}=\sigma\left(\xi_{\sigma}-\widehat{\xi}_{\sigma}-W \operatorname{sign}(\sigma)\right), \\
\dot{V}=\sigma\left(\xi_{\sigma}-\widehat{\xi}_{\sigma}\right)-W|\sigma|, \\
\dot{V} \leq|\sigma| \delta_{0}-W|\sigma| .
\end{gathered}
$$

$\dot{V}$ is strictly negative if $W>\delta_{0}$. Therefore, if $W>\delta_{0}$, locally it creates a sliding regime (see [17]).

\subsection{Observer Assisted Nonlinear Controlled Systems in Input-} Output Representation. In the previous subsection, the power of the GPI observer injections for a proper establishment and development of a first order sliding mode regimen was demonstrated. In this subsection, the GPI observer is used in a wider perspective allowing both sliding surface coordinate function $(\sigma)$ and disturbance function $\left(\xi_{\sigma}\right)$ constructions. These constructions are conducted by means of the system state estimation and disturbance function estimation related to system dynamics; all are supplied by the GPI observer.
Consider the nonlinear, scalar, differentially flat system

$$
y^{(n)}=\psi(t, y) u+\phi\left(t, y, \dot{y}, \ldots, y^{(n-1)}\right)
$$

with the following set of initial conditions: $Y_{0}=\left\{y\left(t_{0}\right)\right.$, $\left.\dot{y}\left(t_{0}\right), \ldots, y^{(n-1)}\left(t_{0}\right)\right\}$. We refer to the function $\psi(t, y)$ as the control input gain of the system. The term $\phi(t, y, \dot{y}, \ldots$, $\left.y^{(n-1)}\right)$ will be addressed as the drift function.

For a given smooth control input function, $u(t)$, let $y(t)=$ $\Theta\left(t, t_{0}, Y_{0}, u(t)\right)$ denote the solution trajectory of system (20) from the set of initial conditions, $Y_{0}$. We denote by the time function $\xi(t)$ the additive disturbance function, regardless of any particular internal structure.

It is desired to drive the flat output $y$ of the system

$$
y^{(n)}=\psi(t, y) u+\xi(t)
$$

to track a given smooth reference trajectory $y^{*}(t)$, regardless of the unknown but uniformly bounded nature of the disturbance function $\xi(t)$. As in the previous case, $\xi$ takes into account, in a lumped way, faults and exogenous and endogenous disturbances affecting the system dynamics. It is important to note that the disturbance functions $\xi$ and $\xi_{\sigma}$ are defined in different dynamics but catch the same essential disturbance behavior. Indeed, it will be showed that it is possible to form an estimate of $\xi_{\sigma}$ from an estimate of $\xi$ and some others estimates provided by the GPI observer.

Regarding controlled system (21), we make the following assumptions.

Assumption 4. The disturbance function $\xi(t)$ is completely unknown, while the control input gain $\psi(t, y)$ is perfectly known. Let $\epsilon$ be a strictly positive real number. The control input gain $\psi(t, y)$ is assumed to be uniformly bounded away from zero; that is, $\inf _{t}|\psi(t, y)| \geq \epsilon>0$ for any solution $y(t)$ of the controlled system. In particular, it is bounded away from zero for the given output reference trajectory $y^{*}(t)$.

Assumption 5. It is assumed that a solution $y(t)$ exists, uniformly in $t$ for every given set of initial conditions $Y_{0}$, specified at time $t=t_{0}$ and for a given, sufficiently smooth control input function $u(t)$. Given a desired flat output reference trajectory $y^{*}(t)$, the flatness of the system, and the previous assumption, a straightforward calculation of the corresponding (unique) open loop control input $u^{*}(t)$ is possible (see [18]).

Assumption 6. Let $m$ be a given integer. As a time function, the $m$ th, time derivative of $\xi(t)$, is uniformly absolutely bounded. In other words, there exists a constant $K_{m}$ so that

$$
\sup _{t}\left|\xi^{(m)}(t)\right| \leq K_{m}
$$

Remark 3. Assumption 6 cannot be verified a priori when $\xi(t)$ is completely unknown. However, in cases where the nonlinearity is known except for some of its parameters, its validity can be assessed with some work. Also, if $\xi^{(m)}(t)$ is not uniformly absolutely bounded almost everywhere, then solutions $y(t)$ for (21) do not exist for any finite $u(t)$ (see [19]). 
2.2.1. Observer-Based Approach. With reference to simplified system (21), in order to propose a GPI observer for a related state and disturbance function estimation, it is considered that the internal model of the disturbance function, $\xi$, is approximated by $d^{m} \xi(t) / d t^{m} \approx 0$ at the observer stage. This model is embedded into the augmented model which is characterized by an extended state composed of the phase variables $x_{1}, x_{2}, \ldots, x_{n}$, associated with the flat output $x_{1}=y$, and augmented by the $m$ output estimation error iterated integral injections $x_{n+1}, \ldots, x_{n+m}$. As a result, setting the state vector $x=\left[\begin{array}{llll}x_{1} & x_{2} & \cdots & x_{n+m}\end{array}\right]$ with $x_{1}=y, x_{2}=\dot{y}, \ldots, x_{n}=y^{(n-1)}$, $x_{n+1}=\xi, \ldots, x_{n+m}=\xi^{(m-1)}$, the augmented state space model is given by

$$
\begin{gathered}
\dot{x}=A x+B \psi(t, y) u+E \xi^{(m)}, \\
y=C x
\end{gathered}
$$

with

$$
\begin{gathered}
A=\left[\begin{array}{ccccc}
0 & 1 & 0 & \cdots & 0 \\
0 & 0 & 1 & & 0 \\
\vdots & & & \ddots & \\
0 & 0 & 0 & \cdots & 1 \\
0 & 0 & 0 & \cdots & 0
\end{array}\right] \in \mathbb{R}^{(n+m) \times(n+m)}, \\
B=\left[\begin{array}{c}
0 \\
\vdots \\
1_{(n \text {th position) }} \\
\vdots \\
0
\end{array}\right] \in \mathbb{R}^{(n+m) \times 1}, \\
C=\left[\begin{array}{llll}
1 & 0 & \cdots & 0
\end{array}\right] \in \mathbb{R}^{1 \times(n+m)}, \quad E=\left[\begin{array}{c}
0 \\
0 \\
\vdots \\
0 \\
1
\end{array}\right] \in \mathbb{R}^{(n+m) \times 1} .
\end{gathered}
$$

Now, the GPI observer for the state, $x$, is proposed:

$$
\begin{gathered}
\dot{\hat{x}}=A \widehat{x}+B \psi(t, y) u+L(y-\widehat{y}), \\
\hat{y}=C \hat{x},
\end{gathered}
$$

where $\hat{x}=\left[\begin{array}{llll}\hat{x}_{1} & \hat{x}_{2} & \cdots & \hat{x}_{n+m}\end{array}\right]^{T}$ is the estimation state vector and the observer gain vector is $L=$ $\left[\begin{array}{llll}l_{n+m-1} & \cdots & l_{1} & l_{0}\end{array}\right]^{T}$.

The estimation error vector, $\widetilde{e}_{x}=\left[\begin{array}{llll}\widetilde{e}_{x 1} & \widetilde{e}_{x 2} & \cdots & \widetilde{e}_{x(n+m)}\end{array}\right]^{T}$, defined as $\widetilde{e}_{x}=x-\widehat{x}$, satisfies

$$
\begin{gathered}
\dot{\tilde{e}}_{x}=(A-L C) \widetilde{e}_{x}+E \xi^{(m)}, \\
\dot{\tilde{e}}_{x}=A_{e} \widetilde{e}_{x}+E \xi^{(m)},
\end{gathered}
$$

where

$$
A_{e}=\left[\begin{array}{ccccc}
-l_{n+m-1} & 1 & 0 & \cdots & 0 \\
-l_{n+m-2} & 0 & 1 & & 0 \\
\vdots & & & & \\
-l_{1} & 0 & 0 & \cdots & 1 \\
-l_{0} & 0 & 0 & \cdots & 0
\end{array}\right],
$$

with $A_{e} \in \mathbb{R}^{(n+m) \times(n+m)}$, and its characteristic polynomial in the complex variable $s$ is given by

$$
\begin{aligned}
p_{e_{x}}(s) & =\operatorname{det}\left(s I-A_{e}\right) \\
& =s^{n+m}+l_{n+m-1} s^{n+m-1}+\cdots+l_{1} s+l_{0} .
\end{aligned}
$$

Theorem 4. Suppose that all previous assumptions are valid. Let the coefficients, $l_{j}$, with $j=0,1, \ldots, n+m-1$, of the polynomial $p_{e_{x}}(s)$ be chosen so that all their roots are exhibited to the left of the complex plane $\mathbb{C}$. Then, the trajectories of the estimation error vector $\tilde{e}_{x}(t)$ globally converge towards a small as-desired sphere of radius $\rho$, denoted by $S(0, \rho)$, centered at the origin of the estimation error phase space $\left\{\widetilde{e}_{x 1}, \widetilde{e}_{x 2}, \ldots, \widetilde{e}_{x(n+m)}\right\}$, where they remain ultimately bounded.

Proof. This problem has already been proposed with slightly different notation in [20]. In a recent work [21], it is shown that the estimation error vector is uniformly ultimately bounded.

Remark 5. Consequently with Theorem 4, the variables $\widehat{x}_{n+1}, \widehat{x}_{n+2}, \ldots, \widehat{x}_{n+m}$ track arbitrarily and closely the unknown time functions $\xi(t)$ and their time derivatives $\xi^{(j)}(t), j=$ $1, \ldots, m-1$.

2.2.2. Sliding Surface Design. Regarding controlled systems (21), a conventional sliding surface can be chosen as (in order to decrease the stable error, an integral term of the tracking error $e_{y}$ can be introduced (see [12]), but it is preferred to maintain a conventional surface to enhance the GPI observer capabilities)

$$
\sigma=e_{y}^{(n-1)}+\lambda_{n-2} e_{y}^{(n-2)}+\cdots+\lambda_{0} e_{y}
$$

with $e_{y}=y-y^{*}$ being flat output tracking error. The design parameters $\lambda_{0}, \ldots, \lambda_{n-2}$ are chosen so that the characteristic polynomial $p_{\sigma}(s)=s^{(n-1)}+\lambda_{n-2} s^{(n-2)}+\cdots+\lambda_{0}$ is Hurwitz. by

An estimated version of the previous surface can be given

$$
\begin{aligned}
\widehat{\sigma}= & \widehat{x}_{n}-\left[y^{*}\right]^{(n-1)}+\lambda_{n-2}\left(\widehat{x}_{n-1}-\left[y^{*}\right]^{(n-2)}\right) \\
& +\cdots+\lambda_{0}\left(\widehat{x}_{1}-y^{*}\right),
\end{aligned}
$$

where $\widehat{y}=\widehat{x}_{1}, \ldots, \widehat{y^{(n-1)}}=\widehat{x}_{n}$ are estimates provided by GPI observer (8). The use of $\widehat{x}_{1}$ instead of $x_{1}$ is preferred for chattering reduction purposes (see [17]).

The sliding surface dynamics of $\widehat{\sigma}$ is given by

$$
\begin{aligned}
\dot{\hat{\sigma}}= & \dot{\hat{x}}_{n}-\left[y^{*}\right]^{(n)}+\lambda_{n-2}\left(\dot{\hat{x}}_{n-1}-\left[y^{*}\right]^{(n-1)}\right) \\
& +\cdots+\lambda_{0}\left(\dot{\hat{x}}_{1}-\dot{y}^{*}\right) .
\end{aligned}
$$




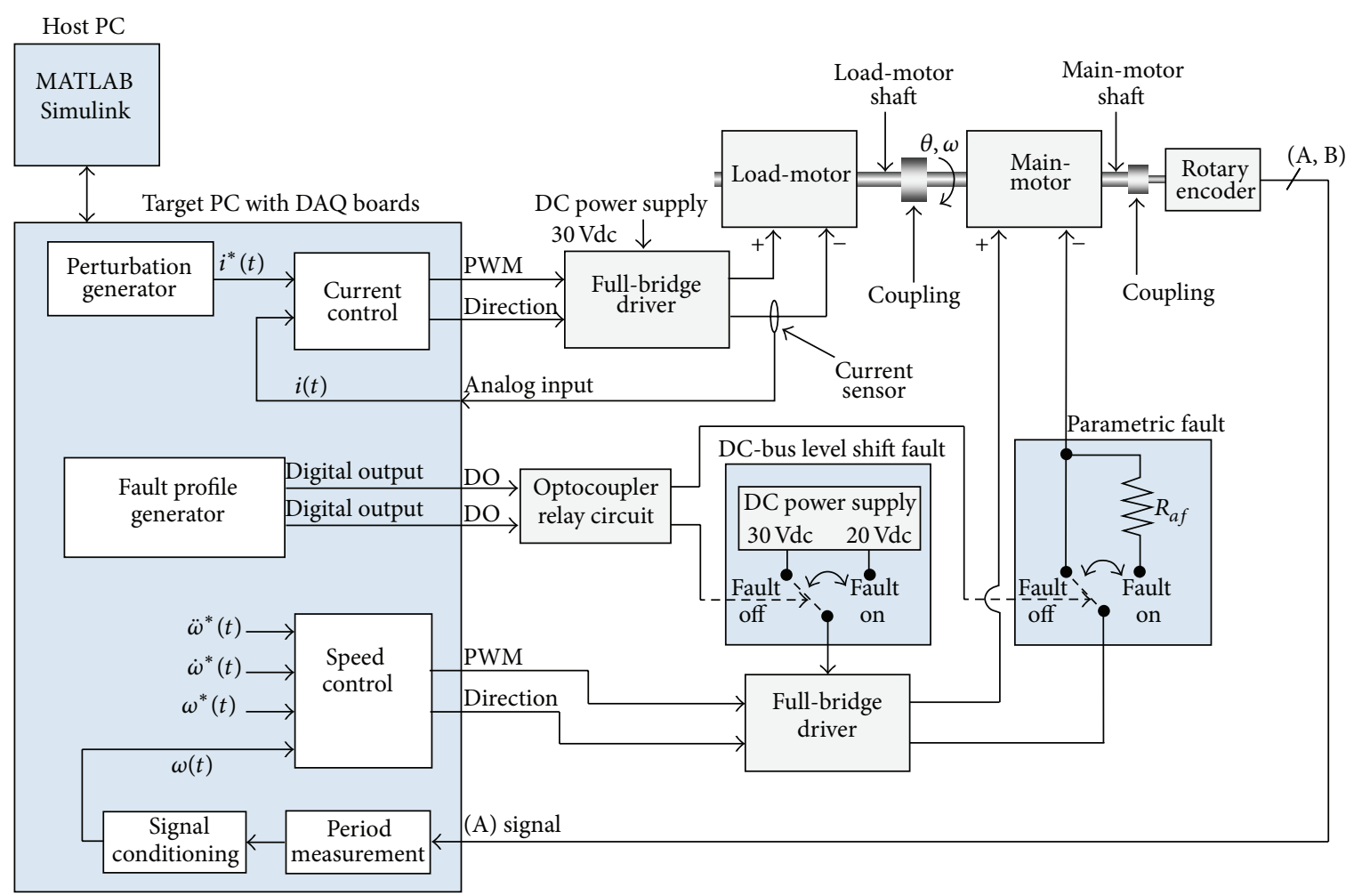

FIGURE 1: General scheme of the experimental setup.

On the other hand from the GPI Observer we have

$$
\dot{\hat{x}}_{n}=\psi(t, y) u+\widehat{x}_{n+1}+l_{m}\left(x_{1}-\widehat{x}_{1}\right),
$$

therefore,

$$
\dot{\hat{\sigma}}=\psi(t, y) u+\widehat{\xi}_{\sigma}
$$

with

$$
\begin{aligned}
\widehat{\xi}_{\sigma}= & \widehat{x}_{n+1}+l_{m}\left(x_{1}-\widehat{x}_{1}\right)-\left[y^{*}\right]^{(n)} \\
& +\lambda_{n-2}\left(\dot{\hat{x}}_{n-1}-\left[y^{*}\right]^{(n-1)}\right)+\cdots+\lambda_{0}\left(\dot{\hat{x}}_{1}-\left[y^{*}\right]^{(1)}\right)
\end{aligned}
$$

where the estimates $\dot{\hat{x}}_{n-1}, \ldots, \dot{\hat{x}}_{1}$ are also provided by the GPI observer. Remember that as stated by the GPI observer state notation $\widehat{x}_{n+1}=\widehat{\xi}$ as announced at the beginning of this subsection, the disturbance function estimation related to sliding regime (33) $\widehat{\xi}_{\sigma}$ is given in terms of $\widehat{\xi}$, which is related to system dynamics (21).

According to (5) the control law is

$$
u=\frac{1}{\psi(t, y)}\left[-\widehat{\xi}_{\sigma}-W \operatorname{sign}(\widehat{\sigma})\right] .
$$

Now, consider the following Lyapunov function candidate:

$$
V_{1}=\frac{1}{2} \widehat{\sigma}^{2}
$$

Differentiating the Lyapunov function (36) with respect to time and using (33) and (35), we obtained

$$
\dot{V}_{1}=\widehat{\sigma} \dot{\widehat{\sigma}}=\widehat{\sigma}\left(\psi(t, y) u+\widehat{\xi}_{\sigma}\right)=-W|\widehat{\sigma}|
$$

which assures the sliding mode regime provided that $W>0$.

\section{Case Study}

The system used for the experimental comparison of the proposed control strategies is a mechatronic system composed of two directly coupled DC motors. The first motor (also called the main-motor) acts as the system to be controlled. The second motor (also called the load-motor) generates perturbation loads to the main-motor. Hence, the proposed control strategies are applied to control the angular speed of the main-motor, while the load-motor acts as the load-torque perturbation generator by means of a current control loop. Figure 1 shows a detailed scheme of the mechatronic system, control loops, and the experimental setup. This figure also shows the implementation scheme of two faults: armature resistance fault (fault 1) and DC-bus level shift fault (fault 2). These faults are typical in systems as the one under study (as it will be explained later), and all controllers will be assessed under these faults.

3.1. Fault-Tolerant Control for DC Motors. The most common faults in DC-motor drives can be classified as actuator, parametric, and sensor faults [22]. The actuator faults are mainly due to the reduction in the performance of amplifiers, 
malfunction of power semiconductors and failures in regulatory stages in the power supply $[23,24]$. Parametric faults are caused by degradation of brushes, inertia and friction changes, and variations in resistance or inductance of the armature [22]. This paper will consider two faults in the mechatronic system:

(1) parametric fault due to a change in the resistance of DC-motor armature and

(2) actuator fault due to level shift of the DC-bus voltage that feeds the full-bridge drive of the main-motor.

3.2. Problem Formulation. Consider the following dynamic model describing a DC-motor controlled by armature voltage $u(t)$, with state variables given by $\omega(t)$ describing the rotor angular speed and $i(t)$ representing the armature current:

$$
\begin{gathered}
L_{a} \frac{d i(t)}{d t}=k_{\mathrm{pwm}} u(t)-R_{a} i(t)-K_{b} \omega(t), \\
J_{m} \frac{d \omega(t)}{d t}=K_{T} i(t)-\tau_{L}(t)-B \omega(t)-\delta(\omega(t)) .
\end{gathered}
$$

The parameters $L_{a}$ and $R_{a}$ represent the armature inductance and armature resistance, $J_{m}$ is the moment of inertia, $K_{b}$ is the back-emf constant, $K_{T}$ is the torque constant, $B$ is the viscous friction coefficient, $k_{\text {pwm }}$ is the conversion gain of PWM, $\tau_{L}$ represents the unknown load-torque perturbation input, $u(t)$ is the control signal, and $\delta(\omega(t))$ represents a nonlinear model of dry friction, where $T_{c}, T_{s}$, and $\alpha$ are terms associated with coulomb friction (see [25]). Consider

$$
\delta(\omega)=\omega\left[>T_{c}\left\{\operatorname{sign}(\omega)+\left(T_{s}-T_{c}\right) e^{-\alpha|\omega|} \operatorname{sign}(\omega)\right\}\right] .
$$

By rewriting and lumping together some terms of (38), the following representation of the system (typical of the ADRC paradigm) is obtained:

$$
\ddot{\omega}(t)=\kappa u(t)+\xi(t),
$$

where

$$
\begin{gathered}
\kappa=\frac{K_{T} k_{\mathrm{pwm}}}{J_{m} L_{a}}, \\
\xi(t)=\frac{K_{T}}{J_{m} L_{a}}\left[-R_{a} i(t)-K_{b} \omega(t)\right] \\
-\frac{1}{J_{m}} \frac{d \tau_{L}}{d t}-\frac{B}{J_{m}} \frac{d \omega(t)}{d t}-\frac{1}{J_{m}} \frac{d \delta(\omega(t))}{d t} .
\end{gathered}
$$

The problem is as follows. Consider a DC-motor described by the dynamics presented in (40), where the rotor angular speed $\omega(t)$ is available for measurement. Given a smooth reference trajectory, $\omega^{*}(t)$, for the angular velocity of the motor shaft, find a control law $u(t)$ such that $\omega(t)$ is forced to track the given reference trajectory $\omega^{*}(t)$. This objective must be achieved even in the presence of unknown disturbances represented by the load input torque $\tau_{L}$, and the effect of parametric and actuator faults.
3.3. Disturbance GPI Observer Design. The following approximation concerning the internal model of the disturbance function $d^{2} \xi(t) / d t^{2} \approx 0$ is considered. According to this approximation, the extended state vector is given by [ $\omega \dot{\omega} \xi \xi]^{T}$ thus we obtain the following augmented plant model:

$$
\left[\begin{array}{c}
\dot{\omega} \\
\ddot{\omega} \\
\dot{\xi} \\
\ddot{\xi}
\end{array}\right]=\left[\begin{array}{llll}
0 & 1 & 0 & 0 \\
0 & 0 & 1 & 0 \\
0 & 0 & 0 & 1 \\
0 & 0 & 0 & 0
\end{array}\right]\left[\begin{array}{c}
\omega \\
\dot{\omega} \\
\xi \\
\dot{\xi}
\end{array}\right]+\kappa\left[\begin{array}{l}
0 \\
1 \\
0 \\
0
\end{array}\right] u+\left[\begin{array}{l}
0 \\
0 \\
0 \\
1
\end{array}\right] \xi^{(2)} .
$$

It is defined as an estimation error: $\widetilde{e}_{\omega}=\omega-\widehat{\omega}$. In order to observe the augmented state, a GPI observer is proposed:

$$
\left[\begin{array}{l}
\frac{d \widehat{\omega}}{d t} \\
\frac{d \hat{\dot{\omega}}}{d t} \\
\frac{d \widehat{\xi}}{d t} \\
\frac{d \dot{\dot{\xi}}}{d t}
\end{array}\right]=\left[\begin{array}{llll}
0 & 1 & 0 & 0 \\
0 & 0 & 1 & 0 \\
0 & 0 & 0 & 1 \\
0 & 0 & 0 & 0
\end{array}\right]\left[\begin{array}{l}
\widehat{\omega} \\
\hat{\dot{\omega}} \\
\widehat{\xi} \\
\hat{\dot{\xi}}
\end{array}\right]+\kappa\left[\begin{array}{l}
0 \\
1 \\
0 \\
0
\end{array}\right] u+\left[\begin{array}{l}
l_{3} \\
l_{2} \\
l_{1} \\
l_{0}
\end{array}\right] \widetilde{e}_{\omega},
$$

where $\left[\begin{array}{llll}l_{3} & l_{2} & l_{1} & l_{0}\end{array}\right]^{T}$ is the observer gains vector. The characteristic polynomial which describes the estimation error dynamics is defined as

$$
p_{\widetilde{e}_{x}}(s)=s^{4}+l_{3} s^{3}+l_{2} s^{2}+l_{1} s+l_{0} .
$$

Given the previously described uncertain model (42), linear GPI observer (43) estimates the augmented state: $[\omega \dot{\omega} \xi \dot{\xi}]^{T}$ with an arbitrary small phase space estimation error, provided that the set of observer design coefficients $\left\{l_{3}, l_{2}, l_{1}, l_{0}\right\}$ is chosen in such a manner that the roots of the characteristic polynomial $p_{\widetilde{e}_{\omega}}(s)$, on the complex variable $s$, are located sufficiently far from the imaginary axis, in the left half side of the complex plane.

3.4. Sliding Control Law Design. By defining the tracking error as $e_{\omega}(t)=\omega(t)-\omega^{*}(t)$, the following sliding surface in terms of the tracking error $e_{\omega}$ are proposed:

$$
\sigma=\dot{e}_{\omega}+\lambda e_{\omega} .
$$

A modified version of the sliding surface that uses estimates of $\omega$ and $\dot{\omega}$ is proposed:

$$
\widehat{\sigma}=\widehat{\dot{\omega}}-\left[\omega^{*}\right]^{(1)}+\lambda_{0}\left(\widehat{\omega}-\omega^{*}\right),
$$

where $\widehat{\dot{\omega}}(t)$ and $\widehat{\omega}(t)$ are estimations provided by a GPI observer (43).

Applying time derivative to (46), the following dynamics is obtained:

$$
\dot{\widehat{\sigma}}=\kappa u+\widehat{\xi}_{\sigma}
$$

with

$$
\widehat{\xi}_{\sigma}=\widehat{\xi}+l_{2}(\omega-\widehat{\omega})-\left[\omega^{*}\right]^{(2)}+\lambda_{0}\left(\frac{d \widehat{\omega}}{d t}-\left[\omega^{*}\right]^{(1)}\right),
$$




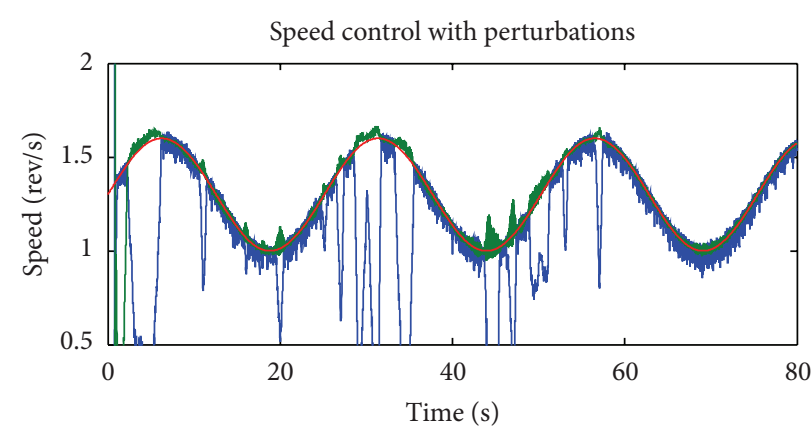

(a)

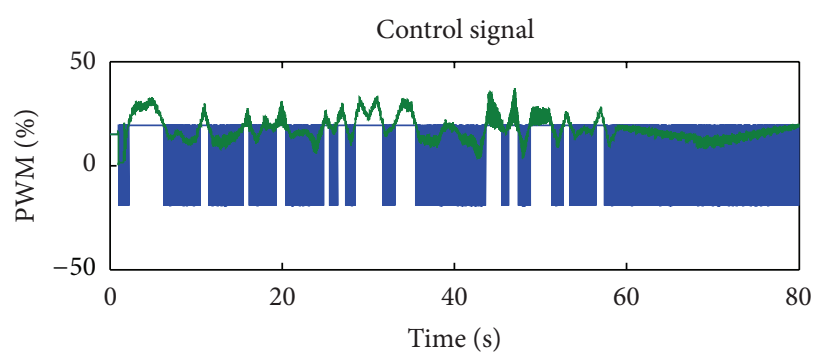

(c)

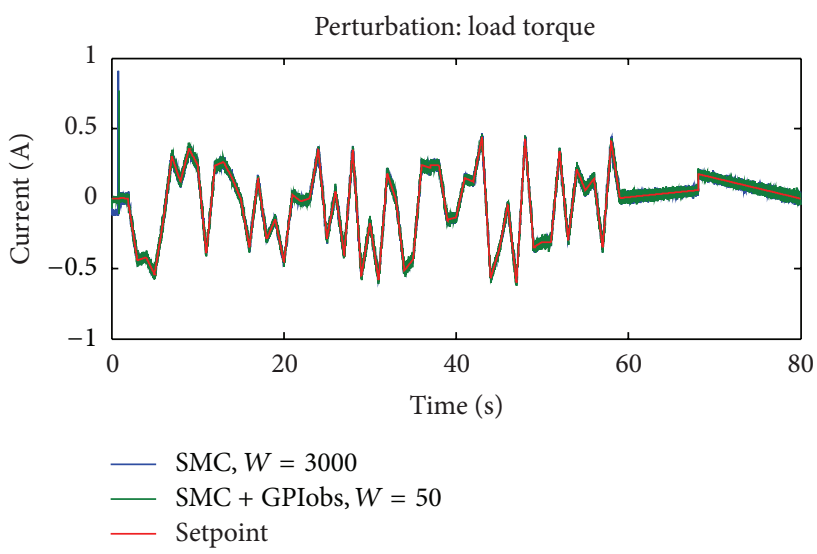

(e)

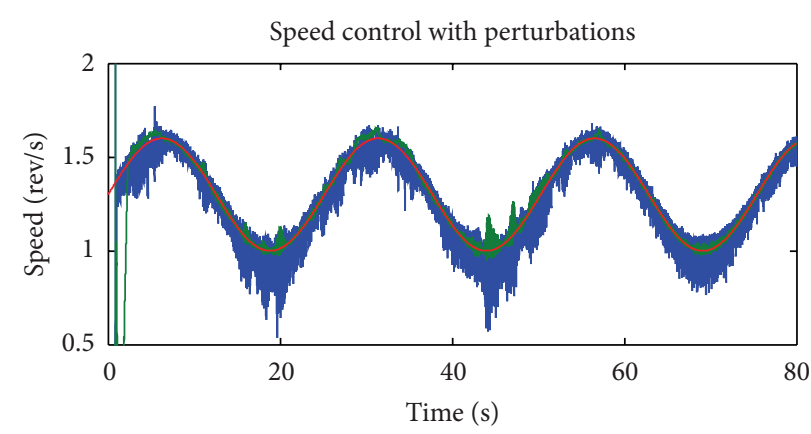

(b)

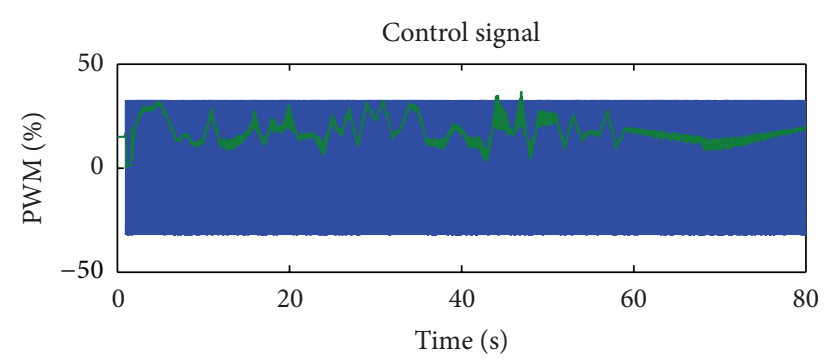

(d)

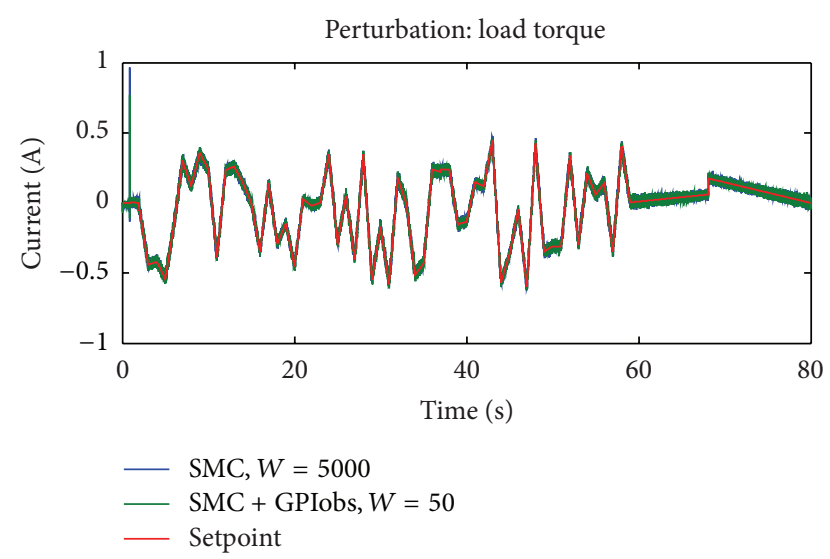

(f)

FIGURE 2: Time response of the control systems under the load-torque perturbations. From top to bottom: speed control response, control signal, and load-torque perturbation.

where the estimates $\widehat{\omega}, \widehat{\xi}$, and $d \widehat{\omega} / d t$ are also provided by GPI observer (43).

Finally, the following discontinuous feedback control law is considered:

$$
u=\frac{1}{\kappa}\left[-\widehat{\xi}_{\sigma}-W \operatorname{sign}(\sigma)\right]
$$

\section{Experimental Results}

In this section, we describe the experiments that were carried out to assess the performance of the proposed GPI observer assisted sliding mode control (SMC+GPIobs) against the classic sliding mode control (SMC) applied to a mechatronic system affected by perturbations and faults. First, the experimental setup is described; then two different operation cases are exposed and analyzed under a tracking problem: system with perturbations and system with faults.

4.1. Experimental Setup. The designed controllers were implemented in a MATLAB xPC Target environment using a sampling period of $0.1 \mathrm{~ms}$ on a computer equipped with a Pentium D processor. The connection between the mechatronic system and each controller was performed by two National Instruments PCI-6024E data acquisition cards. A PWM output at $8000 \mathrm{~Hz}$ and a digital output were both used to command each DC-motor full-bridge driver, one PWM input was used to read the main-motor encoder frequency, two digital outputs were used for enabling/disabling the faults, and an analog input was used to read the load-motor current sensor output. 


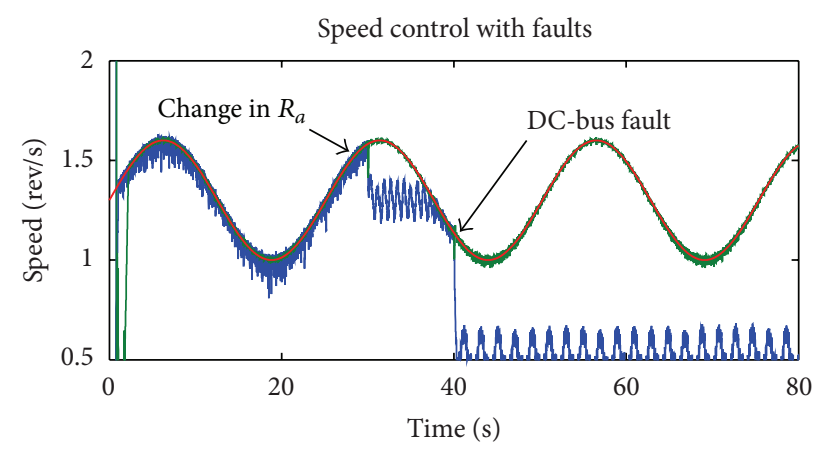

(a)

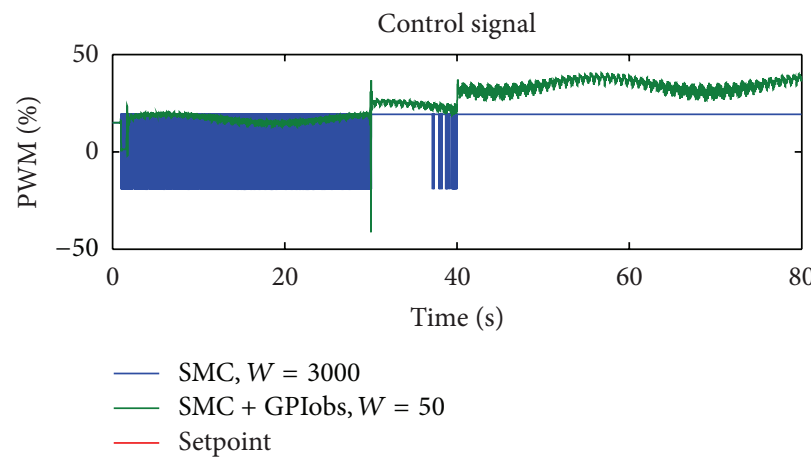

(c)

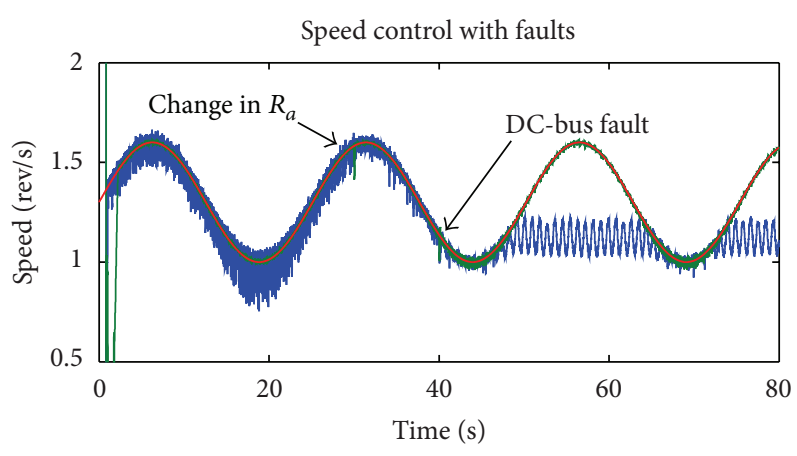

(b)

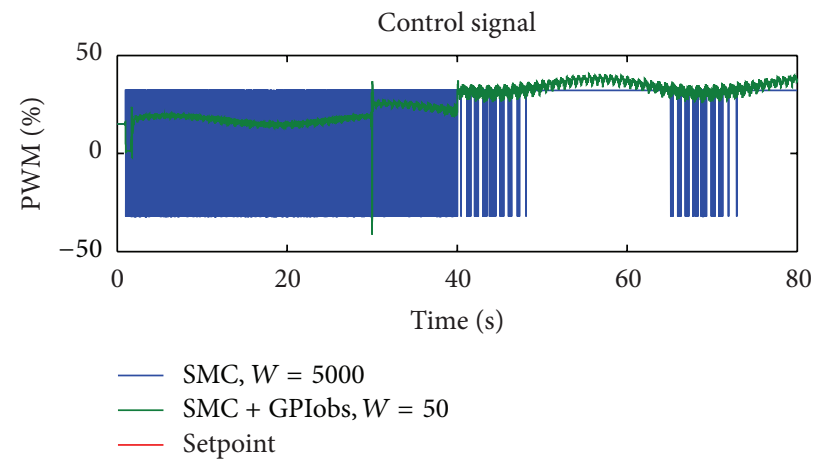

(d)

FIGURE 3: Time response of the control systems under faults 1 and 2. Fault 1 is applied at $t=30$ and fault 2 is applied at $t=40$.

Fault 1 consists of increasing the armature resistance of the main-motor; when this fault is enabled, the armature resistance of the motor is increased by $80 \%\left(R_{\mathrm{af}}=4.7 \mathrm{ohms}\right)$. This fault is enabled (when applicable) at $t=30 \mathrm{sec}$. Fault 2 consists of changing the DC-level of the main-motor full-bridge driver power supply. When this fault goes from disabled to enabled, the DC-level of the power supply changes from $30 \mathrm{Vdc}$ to $20 \mathrm{Vdc}$. This fault is enabled (when applicable) at $t=40 \mathrm{sec}$.

The parameters of the controller are defined as follows: $\kappa=155.6, \lambda_{0}=100, W=50, l_{3}=320, l_{2}=26400, l_{1}=$ 128000 , and $l_{0}=160000$.

4.2. Experimental Results under Perturbations. Figure 2 shows the experimental results of the control systems under evaluation using a setpoint defined as $\omega_{r}^{*}(t)=$ $0.3 \sin (0.25 \pi t)+1.3$. In this case, the control systems are affected by load torque disturbances. The perturbations applied to the main-motor are showed in Figures 2(e) and $2(\mathrm{f})$. Notice that the same perturbation profile is applied to all control schemes in evaluation.

The plots of (a), (c), and (e) in the first column of Figure 2 depict the experiments of the control systems using $W=3000$ for SMC and $W=50$ for SMC+GPIobs. The plots (b), (d), and (f) of Figure 2 in the last column depict the experiments using $W=5000$ for SMC and $W=50$ for SMC+GPIobs. Figure 2(a) shows that the classic SMC (in blue) is highly affected by the perturbations. Although the switching gain was fixed in $W=3000$, the control system gets out of the sliding mode many times and the tracking performance is reduced. Notice that the tracking performance and disturbance rejection may be improved by increasing the switching gain $W$; however, under this condition the chattering phenomenon will be more problematic (see Figure 2(b)).

On the other hand, the proposed GPI observer assisted SMC was capable of rejecting the perturbations and the tracking performance was maintained. In this case, the switching gain $W$ was reduced to $W=50$, which was allowed by the use of the GPI observer that assists the sliding mode controller. Figures 2(c) and 2(d) show that the control signal for SMC+GPIobs (in green) has less control effort than the classic SMC scheme. The chattering in the controlled speed was attenuated even in the presence of unknown perturbations as seen in Figures 2(a) and 2(b) (in green).

4.3. Experimental Results under Faults. Figure 3(a) shows that in presence of fault 1 at $t=30$, the tracking performance of the classic SMC (in blue) is degraded. The selection of $W=3000$ in the classic SMC allows setpoint tracking with no faults; however, it is not possible to maintain the sliding mode for the tracking trajectory when the fault is enabled. Meanwhile, the GPI observer assisted SMC (in green) is capable of tolerating the first fault and even of keeping the tracking performance almost with no change. Note that the switching gain of the proposed strategy is very low ( $W=50$ ), which is beneficial for chattering reduction. It is important to observe that, at 

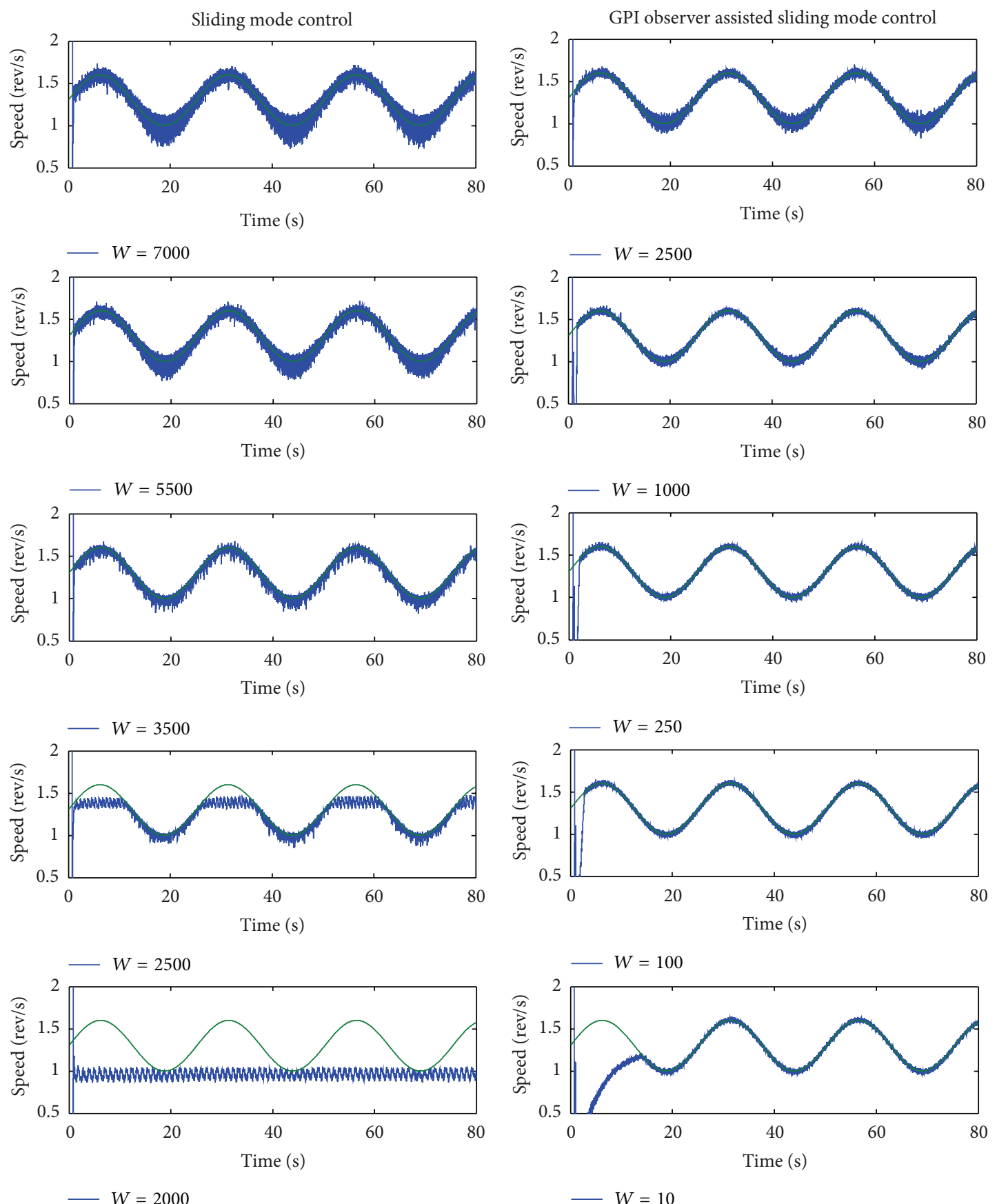

(a)

(b)

FIGURE 4: Time response of the control systems (no faults and no perturbations) under variations in the switching gain $W$. In the first column: sliding mode control; in the second column: GPI observer assisted sliding mode control.

the time of the fault $(t=30)$, there is a tiny transient response in the controlled speed to recover the sliding regime again.

Figure 3(a) also shows the effect of fault 2 in the controlled speed at $t=40$. At this point, it is noticed that after the fault is applied, the classic SMC (in blue) is not capable of maintaining the sliding regime and its tracking performance is highly degraded. Moreover, the GPI observer assisted SMC (in green) tolerates the fault and quickly restores its tracking performance without increasing the chattering.

Figures 3(b) and 3(d) show the experimental results using $W=5000$ for the classic SMC compared to the proposed GPI observer assisted SMC. In this case, the classic SMC can tolerate fault 1 at $t=30$; however, a notable increasing in the chattering is observed. On the other hand, when fault 2 is applied at $t=40$, the classic SMC cannot tolerate it. 
4.4. Chattering Reduction. Figure 4 shows that both control systems in evaluation track the setpoint when no faults and no perturbations are applied. However, the classic SMC requires larger switching gains to track the given setpoint. This issue notably increases the chattering phenomenon in the main-motor controlled speed compared to the proposed GPI observer assisted SMC. In the proposed assisted SMC scheme, the chattering is alleviated by reducing the switching gain down to $W=10$ without loss of the sliding mode. Figure 4 shows that the use of the GPI observer to assist the SMC allows reducing the switching gain $W$; thus the chattering problem is alleviated.

\section{Conclusions}

In this paper, an extension of Generalized Proportional Integral observer-based control has been proposed to the problem of robust creation of sliding regimes for nonlinear singleinput single-output systems, with limited switching control input authority for fault-tolerant operation. The approach considers the use of a GPI observer for the accurate (linear) estimation of nonlinear endogenous, as well as exogenous, disturbance inputs affecting the existence of local sliding regimes on a given smooth sliding manifold. Active, on-line disturbance estimation and subsequent cancellation of statedependent and time-dependent disturbances, significantly contribute to reducing the required switching control amplitude needed to sustain a sliding regime. As an additional bonus it experimented a chattering reduction.

It was shown through the experimental tests that the proposed GPI observer assisted sliding mode control strategy is capable of maintaining the sliding regime even under hard operating conditions such as system uncertainties, perturbations, actuator faults, parametric failures, and small switching control input authority. This demonstrates the robustness of the proposed strategy accomplished by a simple linear GPI observer-based control working on an ADRC paradigm.

It was experimentally probed that (a) the proposed GPI observer-based SMC strategy allows reducing the chattering in the controlled variable (limited by the lumped perturbation estimation error) and (b) the proposed strategy forces the system to approximately keep its nominal performance in the presence of perturbations, faults, and uncertainties.

\section{Acknowledgments}

The authors gratefully acknowledge the support for the realization of this work from the following institutions: Universidad Nacional de Colombia, Cinvestav-IPN, and UPIITAIPN.

\section{References}

[1] C. Edwards, H. Alwi, and C. P. Tan, "Sliding mode methods for fault detection and fault tolerant control," in Proceedings of the 1st Conference on Control and Fault-Tolerant Systems (SysTol '10), pp. 106-117, Nice, France, October 2010.

[2] H. Alwi and C. Edwards, "Fault detection and fault-tolerant control of a civil aircraft using a sliding-mode-based scheme,"
IEEE Transactions on Control Systems Technology, vol. 16, no. 3, pp. 499-510, 2008.

[3] B. Xiao, Q. Hu, and Y. Zhang, "Adaptive sliding mode fault tolerant attitude tracking control for flexible spacecraft under actuator saturation," IEEE Transactions on Control Systems Technology, vol. 20, no. 6, pp. 1605-1612, 2012.

[4] M. T. Hamayun, C. Edwards, and H. Alwi, "Integral sliding mode fault tolerant control incorporating on-line control allocation," in Proceedings of the 11th International Workshop on Variable Structure Systems (VSS '10), pp. 100-105, Mexico City, Mexico, June 2010.

[5] M. Chen and W.-H. Chen, "Sliding mode control for a class of uncertain nonlinear system based on disturbance observer," International Journal of Adaptive Control and Signal Processing, vol. 24, no. 1, pp. 51-64, 2010.

[6] W. Wang and Z.-M. Bai, "Sliding mode control based on disturbance observer for servo system," in Proceedings of the 2nd International Conference on Computer and Automation Engineering (ICCAE '10), vol. 2, pp. 26-29, Singapore, February 2010.

[7] D. Tian, D. Yashiro, and K. Ohnishi, "Improving transparency of bilateral control system by sliding mode assist disturbance observer," IEEJ Transactions on Electrical and Electronic Engineering, vol. 8, no. 3, pp. 277-283, 2013.

[8] J. Yang, S. Li, and X. Yu, "Sliding-mode control for systems with mismatched uncertainties via a disturbance observer," IEEE Transactions on Industrial Electronics, vol. 60, no. 1, pp. 160-169, 2013.

[9] M. Shi, X. Liu, Y. Shi, W. Chen, and Q. Zhao, "Research on the sliding mode based ADRC for hydraulic active suspension of a six-wheel off-road vehicle," in Proceedings of the International Conference on Electronic and Mechanical Engineering and Information Technology (EMEIT '11), vol. 2, pp. 1066-1069, Heilongjiang, China, August 2011.

[10] V. S. Deshpande, M. Bhaskara, and S. B. Phadke, "Sliding mode control of active suspension systems using a disturbance observer," in Proceedings of the 12th International Workshop on Variable Structure Systems (VSS '12), pp. 70-75, 2012.

[11] Y. Cao and X. B. Chen, "Disturbance-observer-based slidingmode control for a 3-dof nanopositioning stage," in Proceedings of the IEEE/ASME Transactions on Mechatronics, vol. PP, no. 99, pp. 1-8, Mumbai, India, 2013.

[12] D.-J. Zhao, Y.-J. Wang, L. Liu, and Z.-S. Wang, "Robust faulttolerant control of launch vehicle via GPI observer and integral sliding mode control," Asian Journal of Control, vol. 15, no. 2, pp. 614-623, 2013.

[13] Z. Gao, Y. Huang, and J. Han, "An alternative paradigm for control system design," in Proceedings of the 40th IEEE Conference on Decision and Control (CDC '01), vol. 5, pp. 4578-4585, December 2001.

[14] G. Tian and Z. Gao, "From poncelet's invariance principle to active disturbance rejection," in Proceedings of the American Control Conference (ACC '09), pp. 2451-2457, June 2009.

[15] J. Han, "From PID to active disturbance rejection control," IEEE Transactions on Industrial Electronics, vol. 56, no. 3, pp. 900906, 2009.

[16] H. Sira-Ramírez and V. F. Batlle, "Robust $\Sigma-\Delta$ modulationbased sliding mode observers for linear systems subject to time polynomial inputs," International Journal of Systems Science, vol. 42, no. 4, pp. 621-631, 2011.

[17] V. Utkin, J. Guldner, and J. Shi, Sliding Mode Control in ElectroMechanical Systems, CRC Press, Boca Raton, Fla, USA, 2nd edition, 2009. 
[18] H. Sira-Ramírez and S. K. Agrawal, Differentially Flat Systems, Marcel Dekker, 2004.

[19] Y. Gliklikh, "Necessary and suffcient conditions for global-intime existence of solutions of ordinary, stochastic, and parabolic differential equations," Abstract and Applied Analysis, vol. 2006, Article ID 39786, 17 pages, 2006.

[20] H. Sira-Ramírez, A. Luviano-Juárez, and J. Cortés-Romero, "Control lineal robusto de sistemas no lineales diferencialmente planos," RIAI: Revista Iberoamericana de Automàtica e Informàtica Industrial, vol. 8, no. 1, pp. 14-28, 2011.

[21] H. Coral-Enriquez, J. Cortés-Romero, and A. G. Ramos, "Robust active disturbance rejection control approach to maximize energy capture in variable-speed wind turbines," Mathematical Problems in Engineering, vol. 2013, Article ID 396740, 12 pages, 2013.

[22] R. Isermann, Fault-Diagnosis Applications, Springer, Berlin, Germany, 2011.

[23] J. H. Lee and J. Lyou, "Fault diagnosis and fault tolerant control of dc motor driving system," in Proceedings of the IEEE International Symposium on Industrial Electronics (ISIE '01), vol. 3, pp. 1719-1723, 2001.

[24] G. Grandi, P. Sanjeevikumar, Y. Gritli, and F. Filippetti, "Faulttolerant control strategies for quad inverter induction motor drives with one failed inverter," in Proceedings of the 20th International Conference on Electrical Machines (ICEM '12), pp. 959-966, 2012.

[25] C. C. de Wit, H. Olsson, K. J. Åström, and P. Lischinsky, "A new model for control of systems with friction," IEEE Transactions on Automatic Control, vol. 40, no. 3, pp. 419-425, 1995. 


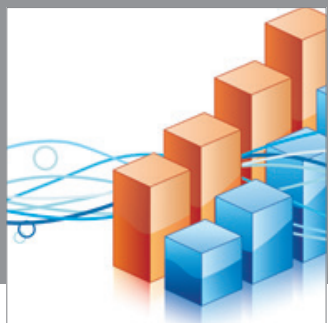

Advances in

Operations Research

mansans

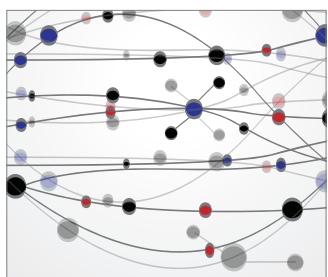

The Scientific World Journal
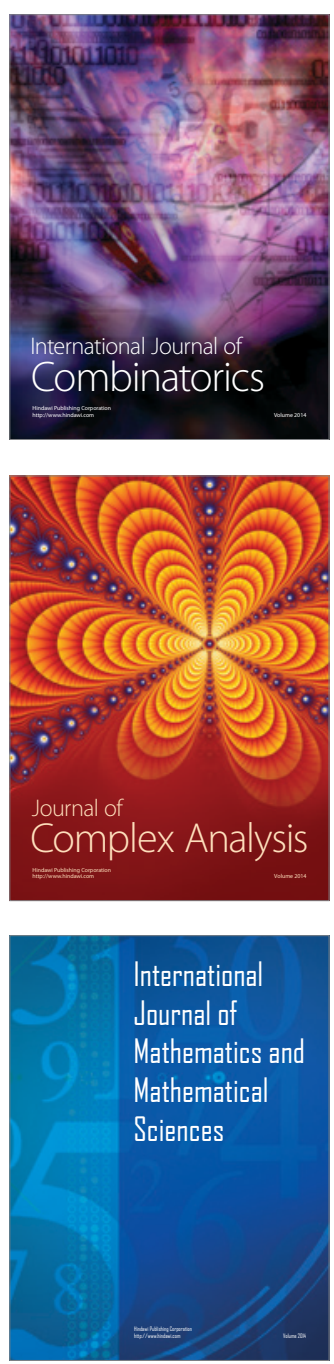
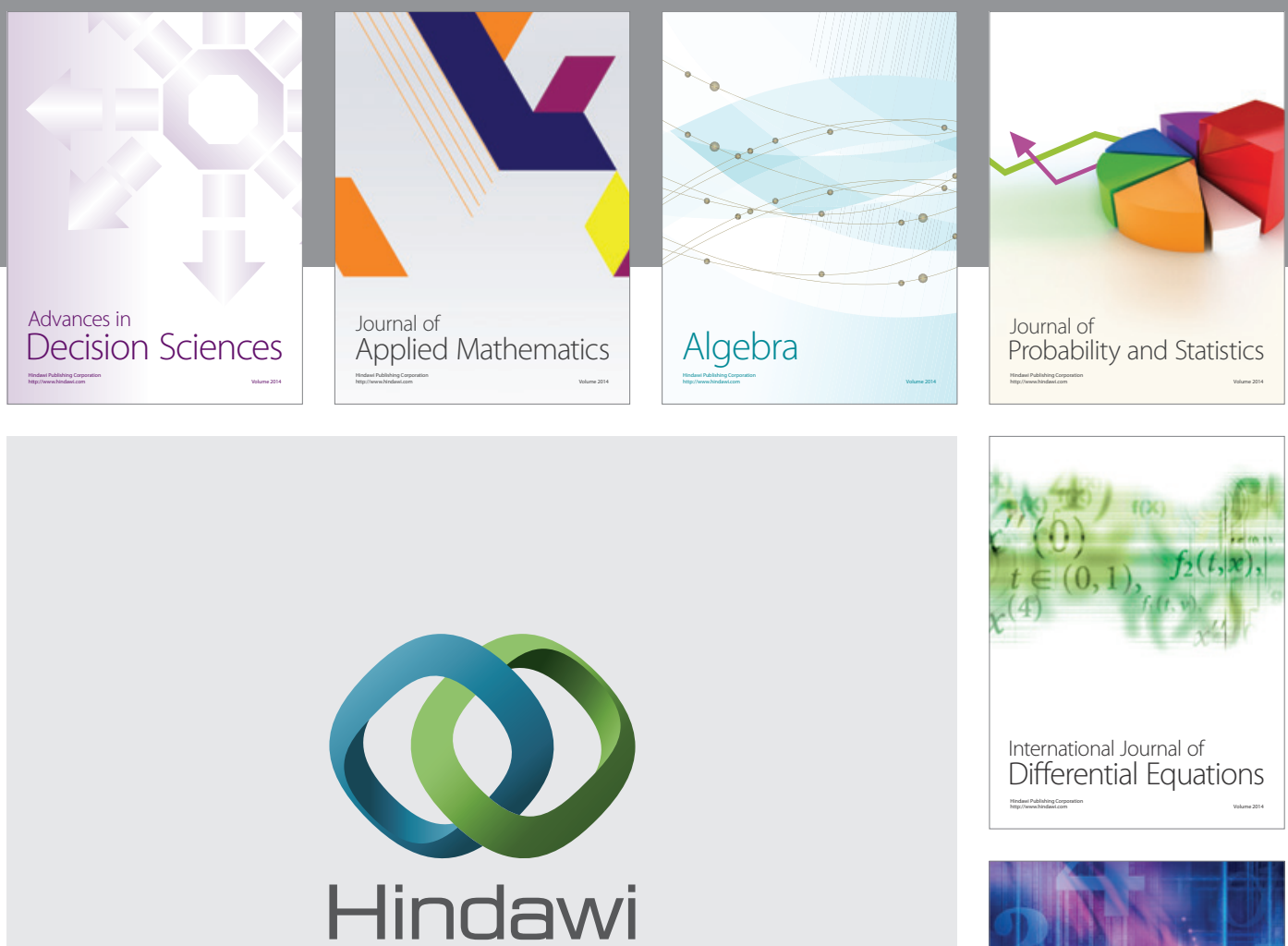

Submit your manuscripts at http://www.hindawi.com
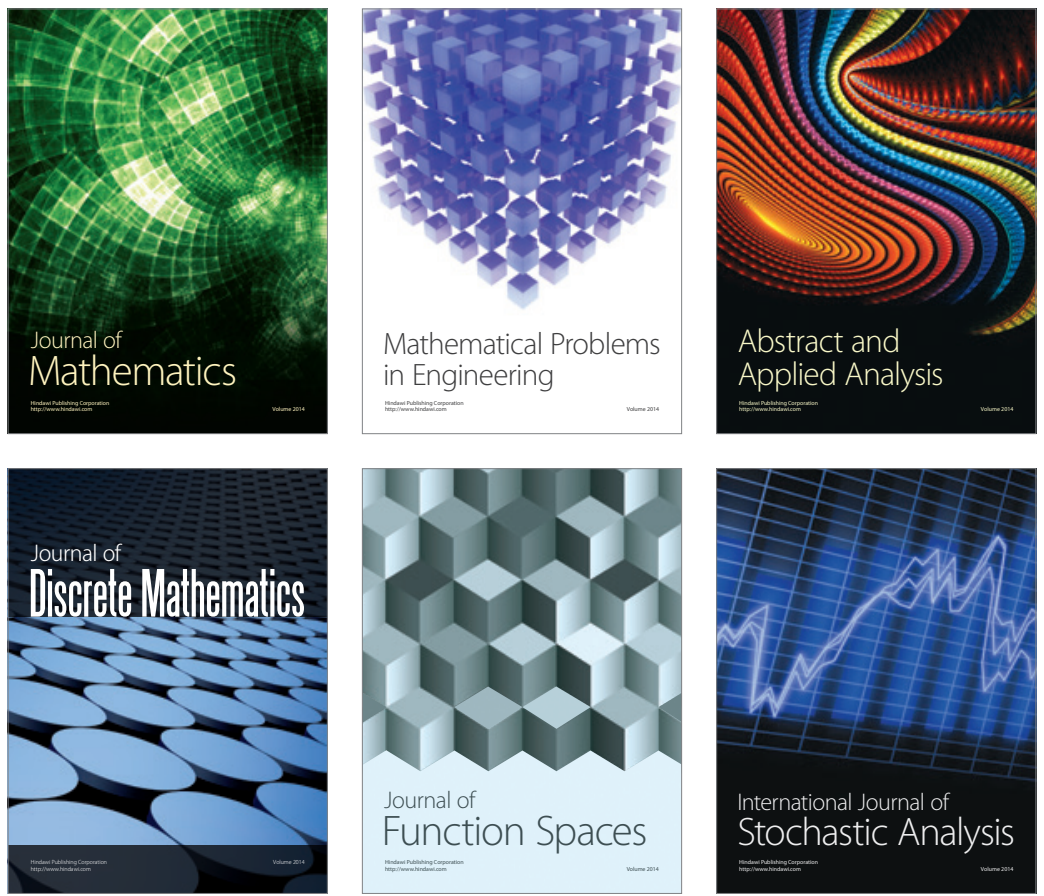

Journal of

Function Spaces

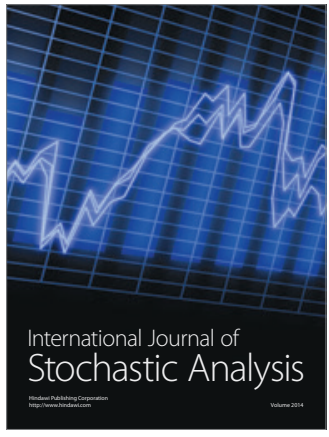

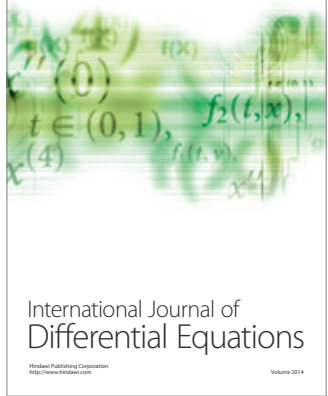
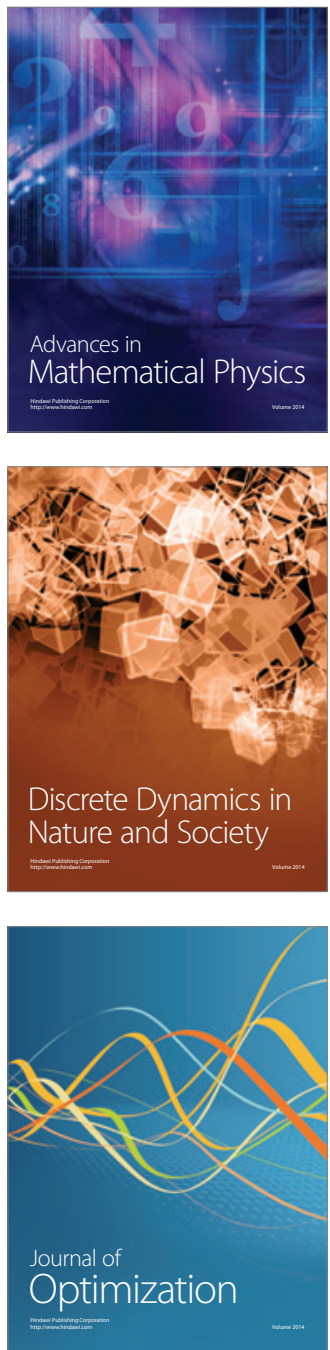